\title{
Earth Orientation since AD 1600
}

\author{
Dennis D. McCarthy \\ U.S. Naval Observatory, Washington, DC 20392, USA
}

\begin{abstract}
Past endeavors in the field of Earth orientation have provided both service to astronomical users of the data and insight into the physical processes of the planet Earth. Since the study of Earth orientation has required a wide geographic distribution of astronomical observing sites, it has been an area of research that has depended heavily on international cooperation over long intervals of time. Recently, IAU Colloquium 178 pointed out that the need exists to find and collect observations that may have been made before the establishment of the current operational services. Reanalysis of such data could be important in our understanding of the physical processes affecting the Earth. All of these observations need to be analyzed in one system consistent with current definitions and conventions. The history of available observations is reviewed along with the contributions that have been made possible using these data. Possible sources for additional data are suggested.
\end{abstract}

\section{Introduction}

Earth orientation refers to the information required to relate a terrestrial reference frame to a celestial reference frame. This includes five quantities: the longitude of the ascending node of the ecliptic, the obliquity of the ecliptic, the $x$ component of polar motion, the $y$ component of polar motion and UT1-UTC, which characterizes the rotation angle of the Earth. The first two quantities describe the motion of the Earth's Celestial Ephemeris Pole in the celestial reference frame, and are provided by the adopted precession/nutation theory plus observational corrections. The second two quantities describe the motion of the Celestial Ephemeris Pole with respect to the adopted terrestrial reference frame. These data can only be obtained through observations. The final quantity describes the angle of rotation of the terrestrial frame with respect to the celestial frame. It too must be observed.

Astronomical observations are required to derive the most accurate theories of precession and nutation and to determine those quantities that cannot be predicted by means of a physical theory. This paper will be concerned only with polar motion and the Earth's rotation angle. The importance of the historical record of these variations and a brief outline of past observations are presented. Only the aspects of polar motion and the Earth's rotation angle that are affected by historical observations will be discussed. Recent high-precision techniques have made it possible to study the high-frequency spectrum of these variations but the historical observations relate only to the lower frequency variations. 


\section{Polar motion}

Polar motion is described by the angular coordinates of the Celestial Ephemeris Pole in a terrestrial reference frame. The origin of the frame in which the polar motion is represented is the north pole of the reference frame. The angular coordinate $x$ is directed along 0 degrees longitude, and the $y$ coordinate is directed along 90 degrees west longitude. Motion of the pole in the terrestrial frame is the result of geophysical and meteorological phenomena. Reviews of the subject can be found in Munk \& McDonald (1960), Lambeck (1980), Rochester (1973), and Eubanks (1993).

The International Latitude Service (ILS) was organized in 1892 to make astronomical observations of polar motion by observing the variation of astronomical latitude at the participating observatories. These observations began in late 1899. IAU Colloquium 178 was held in 1999 to mark the centennial of its founding. The proceedings (Dick et al. 2000) contains a comprehensive review of the history of the ILS as well as other international organizations that have been concerned with the determination of polar motion.

The observations of the ILS along with others made since 1900 have recently been re-analyzed (Vondrák et al. 1997; Vondrák, 1999) to provide a consistent set of data referred to the most current realization of the International Earth Rotation Service (IERS) reference frames. The accuracy of the observations has improved by more than a factor of 1000 since the beginning of the ILS.

The observations have established that the pole experiences motions over a wide range of time scales. Only the lower frequency variations will be discussed here since the historical observations are used mainly to improve our knowledge of polar motion with longer time scales.

Secular polar motion is the apparently non-periodic motion of the pole along the meridian of 70 degrees west longitude by approximately 3 mas per year. For some time the reality of this motion was questioned because of the possibility of systematic errors in the star proper motions. However, the reanalysis by Vondrák and his colleagues (Vondrák et al. 1997; Vondrák 1999) using Hipparcos proper motions has proved the motion to be real. The most recent high-accuracy observations also agree that the current motion is consistent with the historical motion. This motion is most likely due to the effects of post-glacial rebound, a phenomenon that is due to the motion of the Earth's crust caused by the uplift following the melting of glaciers. Other possible contributors to this motion include variations in continental water storage.

The Celestial Ephemeris Pole also experiences motions in the terrestrial frame on decadal time scales. These are poorly understood and are thought to be due possibly to changes in ground water storage, changes in glacial ice or changes in sea level. Perhaps the most enigmatic of the polar motions is the Chandler motion with a period of approximately 433 days. This is due to the free motion of the pole caused by the fact that the axis of rotation is not collinear with respect to one of the principal axes of inertia. This is a damped motion whose excitation is poorly understood.

Historical observations have been instrumental in establishing the nature of these variations. They have also raised questions that remain unanswered. These observations have been based largely on the ILS observations. However 
there have been significant contributions from observatories not connected to the ILS. Many such observations remain unanalyzed for their possible contribution to the study of polar motion. They were made mainly in connection with observational programs to observe aberration and/or nutation or to establish stellar reference frames. However, it is possible that analyses of these observations could provide observations of the motion of the pole that, even with their relatively low accuracy could provide useful information, particularly for the low-frequency components of polar motion.

\section{Rotation Angle}

As is the case with polar motion, the Earth's rotation angle is subject to a wide variety of motions. The tidal interaction with the Moon causes a secular deceleration of the Earth's rotation. Decadal variations are also evident.

Observations of the Earth's rotation depend on ancient eclipse records, lunar occultations and in recent times measurements that were made specifically to measure the Earth's rotation angle.

Since the advent of the telescope in the early seventeenth century the principal contribution until the mid twentieth century has been lunar occultations. See Stephenson (1997) for a comprehensive review of the subject.

All of these observations provide an estimate of $\Delta \mathrm{T}$, the difference between the time determined by the variable rotation of the Earth and a uniform time scale. Before the advent of uniform atomic time, this time scale was provided by Ephemeris Time defined by the orbital motion of the Earth and determined from lunar observations. Atomic time now provides the uniform time scale. The obvious general characteristic of $\Delta \mathrm{T}$ is the parabolic nature of the curve caused by the apparently secular decrease in the length of the day. The principal cause of this phenomenon is the tidal deceleration, but there is a significant nontidal component attributed to the changing shape of the Earth's gravity field due to postglacial rebound. This information is used to calibrate and check viscoelastic rebound models of the Earth's crust. Also evident on this plot are the decadal fluctuations that are apparently the result of interactions between the core and mantle of the Earth. The nature of the core-mantle coupling and the possible ocean/atmosphere interaction with the solid Earth at these frequencies remains unsolved.

\section{What Do We Know As A Result of Historical Observations?}

Historical observations of Earth orientation have made important contributions to our understanding of the Earth and the establishment of operational reference systems. They have established the reality of the apparently secular motion of the celestial ephemeris pole in the terrestrial reference frame. It is most likely the result of post-glacial rebound. The decadal polar motion with variations occurring on time scales of twenty to thirty years has also been established, but the cause remains unknown. Historical observations have also contributed to the study of the Chandler motion of the pole. Although the annual elliptical motion of the pole is now known to be driven meteorologically, the variations in 
amplitude and phase remain enigmatic and these observations are invaluable in the study of possible excitation and damping mechanisms.

Historical observations of the Earth's rotation have established the nature of the secular change in length of day due mainly to tides. The decadal variations have been linked to magnetic field variations and the annual variation is clearly of meteorological origin

In geodesy, geophysics, and timekeeping these historical observations have contributed, and continue to contribute, to our understanding of core-mantle coupling, precise geodetic reference frames, atmosphere-solid Earth interactions, plate motion, post-glacial rebound, lunar studies, tides, Love numbers, and the definition of the second.

\section{New Contributions of Historical Data}

IAU Colloquium 178 (Dick et al. 2000) pointed out that the need exists to find and collect observations that may have been made before the establishment of the modern operational services. Reanalysis of such data could be important in our understanding of the physical processes affecting the Earth. All of them need to be analyzed in one system consistent with current definitions and conventions. New sources of possibly relevant data should be investigated including past local observations of "variation of astronomical latitude" and occultation observations. Particularly important to these analyses is the evaluation of their systematic errors.

\section{References}

Dick, S., McCarthy, D.D., Luzum, B., (eds) 2000, Polar Motion: Historical and Scientific Problems, (San Francisco: ASP Conference Series, 208)

Eubanks, T.M. 1993, "Variations in the Orientation of the Earth," in Contributions of Space Geodesy to Geodynamics: Earth Dynamics, (Washington, DC: American Geophysical Union)

Lambeck, K. 1980, The Earth's Variable Rotation (Cambridge: Cambridge University Press)

Munk, W.H., \& MacDonald, G.J.F. 1960, The Rotation of the Earth (Cambridge: Cambridge University Press)

Rochester, M. 1973, "The Earth's Rotation", EOS, Transactions American Geophysical Union, 54, pp.769-781

Stephenson, F.R. 1997, Historical eclipses and earth's rotation (Cambridge: Cambridge University Press)

Vondrák, J., Ron, C., \& Pešek, I. 1997, "Earth rotation in the Hipparcos reference frame", Cel. Mech. and Dynam. Astron., 66, 115-122

Vondrák, J. 1999, "Earth Rotation Parameters 1899.7-1992.0 after Reanalysis within the Hipparcos Frame", Surv. Geophys, 20, 165-195 